\title{
Size and Albedo of the Object of Interstellar Origin A/2017U1
}

\author{
N. Chandra Wickramasinghe ${ }^{1,2,3}$, Janaki T. Wickramasinghe ${ }^{1}$, Daryl H. Wallis \\ ${ }^{1}$ Buckingham Centre for Astrobiology, University of Buckingham, UK \\ ${ }^{2}$ Centre for Astrobiology, University of Ruhuna, Matara, Sri Lanka \\ ${ }^{3}$ Institute for the Study of Panspermia and Astrobiology, Gifu, Japan \\ Email: ncwick@gmail.com
}

\begin{abstract}
The interstellar object A/2017U1 has been reported to possess a highly elongated shape with a characteristic average radius of $102 \mathrm{~m}$. We argue that the evolution of an organic-rich cometary surface in interstellar space could lead to the formation of an aerogel-like surface with an albedo of 0.0005 leading to a more realistic characteristic dimension of $\sim 1000 \mathrm{~m}$.
\end{abstract}

Keywords: Comets, albedo, size

\section{Introduction}

The discovery by Meech et al. (2017) [1] of the first interstellar cometary or asteroidal body entering the solar system on a hyperbolic orbit leads to several interesting problems [2]. Its observed behaviour near perihelion, including a periodic intensity variation of amplitude $\sim 2.5 \mathrm{mag}$ has led to the conclusion that the object is a highly elongated oblate spheroid with axial ratio 10:1. A notional average radius for this elongated object is given by Meech et al. as $102 \mathrm{~m}$, considerably smaller than any typical comet. This value of average radius is based on assuming the cometary surface material to have an albedo 0.04 , typical of comets. The possibility of the cometary surface material possessing a much lower albedo/reflectivity at visible wavelengths cannot be excluded and will now be explored.

\section{Low Albedo Comets}

Ever since the Giotto mission to Comet P/Halley in 1986 cometary surfaces of low albedo, typically 0.04 , have come to be regarded as commonplace. Values of albedo in the range $\sim 0.01-0.04$ seem appropriate for most comets including Comet Tempel 1 and Comet 67P/C-G although dark spots on Comet Borrelly have been found to have an albedo $0.008[3,7]$. It has also been argued that a very large class of low albedo comets exist that are yet unobserved (J.T. Wickramasinghe)[4].

Recent studies of comets point to the existence of surface material dominated by organic molecules, which are most likely associated with biology. Such cometary surfaces after exposure to the conditions of interstellar space for millions of years would be expected to undergo chemical and structural evolution leading to increased porosity and the development of vacuous fairy castle or aerogel like structures.

\section{Reflectivity of an Exceedingly Porous Organic Regolith}

It is of interest in this connection that particles of probable cometary origin entering the ionosphere and stratosphere have a fluffy, porous structure, with vacuum filling factors $\sim 0.75-0.95$ (Rietmeijer, 2002) [5]. A tarry medium of refractive index $m_{1}=n_{1}-i k_{1}$, within which are distributed vacuum spheres of refractive index 1 (porosity) occupying a fraction $f$ of the total volume of material, behaves as one of complex refractive index $m$ given by

$$
m^{2}=m_{1}^{2}\left[1+\frac{3 f\left(1-m_{1}^{2}\right) /\left(1+2 m_{1}^{2}\right)}{1-f\left(1-m_{1}^{2}\right) /\left(1+2 m_{1}^{2}\right)}\right]
$$

where $m=n-i k, n, k$ being the real and imaginary refractive indices (Bohren and Wickramasinghe, 1977) [6]. Assuming normal incidence the reflectivity $R$ of a plane slab of this material is given by the formula from classical electromagnetic theory. 


$$
R=\frac{(n-1)^{2}+k^{2}}{(n+1)^{2}+k^{2}}
$$

For a tarry organic fairy-castle-like matrix and with given values of the vacuum volume fraction $f$ (porosity of the aerogel structure) we can readily solve for $m(=n-i k)$ from (1), and thus obtain the effective reflectivity $R$, which is the effective albedo $(=p)$ of the cometary material. If we now take $n=$ 1.45 , and $k=0-0.1$ at optical wavelengths, as being representative of organic polymers (e.g. kerogen), the resulting values of $R$ are displayed in Fig. 1 .

It is evident that very low values of reflectivity $R(=p)$ can be obtained with vacuum fractions that are in line with existing estimates for loose aggregations of interstellar dust grains: the bulk porosities $(=f)$ of interplanetary dust particles are $\sim 0.75$ and those of Type III fireballs of cometary origin are $\sim 0.95$ (Rietmeijer, 2002) [5]. A very low albedo $\mathrm{R}$ can be achieved when a comet's already porous surface loses ice and volatile organics between the interstices. In due course with the depletion of volatiles the cometary surface becomes an aerogel, and the albedo rapidly falls to very low values $p$ $<<0.001$ (Fig. 1).

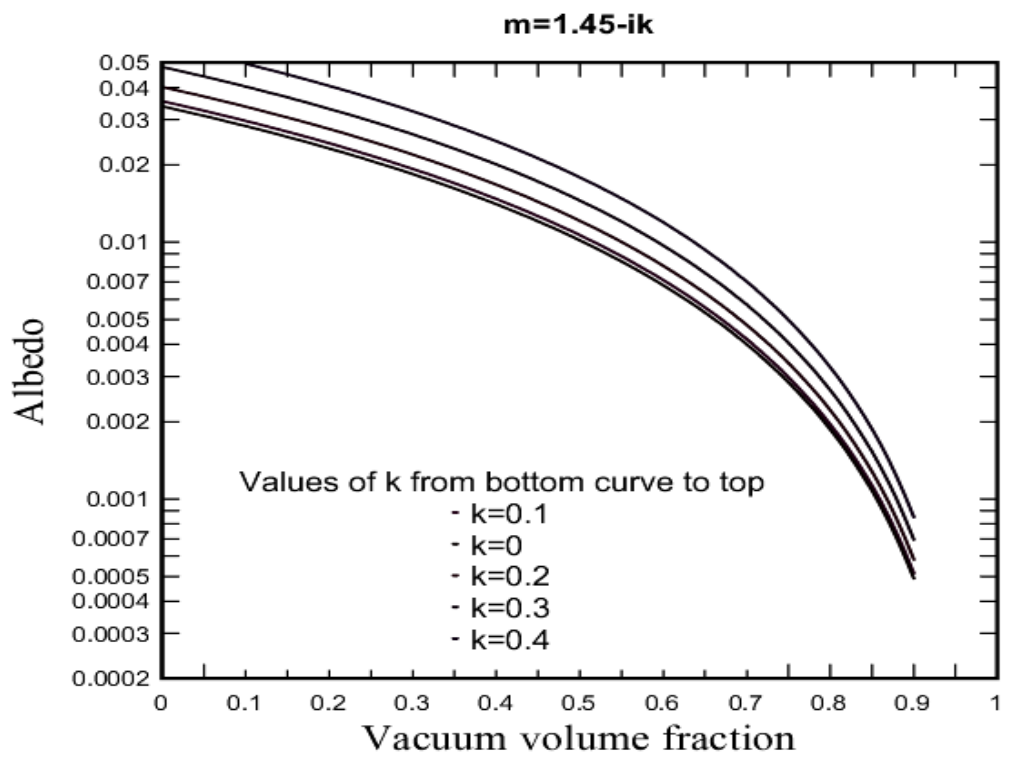

Figure 1. The reflectivity of an aggregate of organic particles $10^{-5} \mathrm{~cm}$ in diameter as a function of porosity. For comparison, the nuclei of active comets have albedos $p \approx 0.02-0.04$, while dark spots on comet Borrelly have $p \approx$ 0.008 (Nelson et al.., 2004) [7].

\section{Application to $\mathrm{A} / 2017 \mathrm{U} 1$}

We now consider the implications of assuming the surface of A/2017U1 to be of a similar aerogel-like material. If $R$ is the effective radius and $p$ is its albedo then for a given value of its brightness the quantity $R^{2}$ p remains constant. Thus equating with flux expected from the notional radius of $102 \mathrm{~m}$ with albedo 0.04 given by Meech et al. (1) we have

$$
R^{2} p=0.04 \times(102)^{2}
$$

Giving

$$
R=102 \times(0.04 / p)^{1 / 2}
$$

With $p=0.0005$ appropriate for a $90 \%$ vacuum volume fraction (Fig.1) we get $R=912 \mathrm{~m}$. This gives a more realistic model with notional cometary spheroid of semi major axis of the order of several $\mathrm{km}$.

We conclude by remarking that with reasonable assumptions about the interstellar evolution a Halleytype or $67 \mathrm{P} / \mathrm{C}-\mathrm{G}$ type cometary surface in interstellar space, we could obtain a low enough albedo for A/2017U1 so as to be consistent with a "standard" solar system-type comet. 


\section{References}

1. Meech, K.J., Weryk, R., Micheli, M. et al., (2017). Discovery and characterisation of the first known interstellar object, Nature, November 2017

2. Wickramasinghe, N.C. et al. (2018) "Oumuamua (A/2017U1)-A Confirmation of Links between Galactic Planetary Systems," Advances in Astrophysics, 3(1): 43-46.

3. Wickramasinghe, J.T., Wickramasinghe, N.C. and Napier, W.M., (2013) Comets and the Origin of Life (World Scientific Press)

4. Wickramasinghe, J.T., (2007). The role of comets in panspermia (Cardiff University PhD thesis)

5. Rietmeijer, F. J. M., (2002). In: E. Murad and I.P. Williams, eds. Meteors in the Earth's Atmosphere. Cambridge: Cambridge Univ. Press, pp. 215.

6. Bohren, C. F., Wickramasinghe, N. C., (1977). On the computation of optical properties of heterogeneous grains. Astrophys. and Space Sci., 50, pp. 461-472.

7. Nelson, R.M., Soderblom, L.A., Hapke, B.W. (2004) Are the circular dark features on Comet Borrelly's surface albedo variations or pits? Icarus, 167, 37 . 